\title{
Neutrino spectra from stellar electron capture
}

\author{
K. Langanke ${ }^{1}$, G. Martínez-Pinedo ${ }^{1,2}$ and J. M. Sampaio ${ }^{1}$ \\ ${ }^{1}$ Institut for Fysik og Astronomi, Århus Universitet, DK-8000 Arhus C, Denmark \\ ${ }^{2}$ Departement für Physik und Astronomie, Universität Basel, Basel, Switzerland
}

(November 6, 2018)

\begin{abstract}
Using the recent shell model evaluation of stellar weak interaction rates we have calculated the neutrino spectra arising from electron capture on $p f$-shell nuclei under presupernova conditions. We present a simple parametrization of the spectra which allows for an easy implementation into collapse simulations. We discuss that the explicit consideration of thermal ensembles in the parent nucleus broadens the neutrino spectra and results in larger average neutrino energies. The capture rates and neutrino spectra can be easily modified to account for phase space blocking by neutrinos which becomes increasingly important during the final stellar collapse.
\end{abstract}

PACS numbers: $26.50 .+\mathrm{x}, 23.40 .-\mathrm{s}, 21.60 . \mathrm{Cs}$

Weak interactions play a central role in the final evolution of massive stars [1]. These processes, mainly electron capture and beta decays, create neutrinos which for all densities until the collapse becomes truly hydrodynamic (i.e. $\rho \sim 10^{11} \mathrm{~g} \mathrm{~cm}^{-3}$ ) escape the star carrying away energy and reducing the entropy. Thus, in simulations which follow the star's evolution until the iron core reaches central densities of order a few $10^{9} \mathrm{~g} \mathrm{~cm}^{-3}$ it is sufficient to know the average neutrino energies of the various weak processes to determine the energy loss rate along the stellar trajectory, e.g. [2]. Such simulations define the 'presupernova models', e.g. 3], which are then being used as input to detailed studies of the collapse and explosion mechanism, e.g. 四. In recent years it has become apparent that the various neutrino reactions play an essential role in these simulations and, as neutrino reactions depend sensitively on energy, these studies require a detailed bookkeeping of the neutrino spectra. This goal is achieved within hydrodynamical models with explicit neutrino Boltzmann transport [5]. The necessary input into such simulations are the weak rates and the associated neutrino spectra.

So far, electron capture on nuclei has only been treated quite schematically in collapse simulations with neutrino Boltzmann transport, e.g. [5]. The ensemble of nuclei in the stellar composition, given by nuclear statistical equilibrium, is represented by an 'average nucleus', whose capture rate then is derived on the basis of the independent particle model (even reduced to a model which only considers $f_{7 / 2}$ and $f_{5 / 2}$ orbitals [5]). The corresponding neutrino spectrum is then approximated in the spirit of this model, assuming a nucleus-independent energy splitting of $3 \mathrm{MeV}$ between the $f_{7 / 2}$ and $f_{5 / 2}$ orbitals. We note, that the standard for the stellar weak interaction rates in presupernova evolutions, however, was set by the work of Fuller, Fowler and Newman (FFN) [8,9] who estimated the rates for the nuclei with mass numbers $A=21-60$ on the basis of the independent particle model and experimental data, whenever available. Presupernova evolution studies then considered the FFN rate tables for a proper nuclear composition. FFN list the average neutrino energies for the various weak reac- tions, but to our knowledge neutrino spectra have not been derived on the basis of the FFN compilation. As mentioned above, such spectra are also not required for presupernova studies as the neutrinos leave the star unhindered.

Due to progress in nuclear many-body modeling and in computer hardware and guided by experimental data it has recently been possible to treat the nuclear structure problem involved in the calculation of stellar weak interaction rates in a reliable way [10,11]. The calculations have been performed on the basis of large-scale shell model studies which reproduce all experimentally available relevant data quite accurately $[12]$. These shell model rates show some marked differences to the FFN estimates, leading to significant changes in the presupernova evolution of massive stars [2,13]. It appears therefore reasonable that this compilation [11] should also be used in collapse and explosion studies which build on the presupernova models. To make such use possible we will here study the neutrino spectra corresponding to the shell model rates and show a way how these spectra can be easily and consistently implemented in collapse codes. It is important to note that under the conditions of the presupernova models and in the subsequent stellar evolution beta decay is strongly blocked by the appreciable electron chemical potential and the total electron capture rates are orders of magnitude larger [10,2,13]. Thus it is quite sufficient to focus on the neutrino spectra arising from electron captures for post-presupernova simulations where detailed neutrino transport is important.

Under the stellar conditions we are concerned with electron capture is dominated by Gamow-Teller (GT) transitions [14]. The appropriate formalism has been derived in 8,90 :

$$
\begin{aligned}
\lambda & =\frac{\ln 2}{K}\left(\frac{g_{A}}{g_{V}}\right)^{2} \sum_{i, j} \frac{\left(2 J_{i}+1\right) e^{-E_{i} /(k T)}}{G(Z, A, T)} \frac{\left|\left\langle j|| \sum_{k} \sigma^{k} t_{+}^{k} \| i\right\rangle\right|^{2}}{2 J_{i}+1} \\
& \times \int_{w_{l}}^{\infty} w p\left(Q_{i j}+w\right)^{2} F(Z, w) S_{e}(w) d w
\end{aligned}
$$

where the sums in $i$ and $j$ run over states in the parent and daughter nuclei, respectively. For the constant $K$ 
we used $K=6146 \pm 6 \mathrm{~s}$ [15 and $g_{V}, g_{A}$ are the vector and axialvector coupling constants. $G(Z, A, T)=$ $\sum_{i} \exp \left(-E_{i} /(k T)\right)$ is the partition function of the parent nucleus. The sum in the GT matrix element runs over all nucleons. In the phase space integral $w$ is the total energy of the electron in units of $m_{e} c^{2}$, and $p=\sqrt{w^{2}-1}$ is its momentum in units of $m_{e} c$. Finally the Q-value for a transition between two nuclear states $i, f$ is defined in units of $m_{e} c^{2}$ as

$$
Q_{i j}=\frac{1}{m_{e} c^{2}}\left(M_{p}-M_{d}+E_{i}-E_{j}\right),
$$

where $M_{p}, M_{d}$ are the nuclear masses of the parent and daughter nucleus, respectively, while $E_{i}, E_{j}$ are the excitation energies of the initial and final states. The lower integral limit in (1) is $w_{l}=1$ if $Q_{i j}>-1$ or $w_{l}=\left|Q_{i j}\right|$ if $Q_{i j}<-1$. For the stellar conditions we are interested in, the electrons are well described by Fermi-Dirac (FD) distributions, with temperature $T$ and chemical potential $\mu_{e}$ :

$$
S_{e}\left(E_{e}\right)=\frac{1}{\exp \left(\frac{E_{e}-\mu_{e}}{k T}\right)+1},
$$

with $E_{e}=w m_{e} c^{2}$. The remaining factor appearing in the phase space integrals is the Fermi function, $F(Z, w)$, that corrects the phase space integral for the Coulomb distortion of the electron wave function near the nucleus.

Applying the formalism described above and determining the nuclear matrix elements within large-scale shell model calculations, stellar electron capture rates have recently been calculated for $p f$-shell nuclei. These nuclei dominate the weak processes in the presupernova evolution of massive stars and details of the rate evaluations can be found in 10]. Here we will use the same approach to study the neutrino spectra emerging in the electron capture reactions. Energy conservation requires that the neutrino emitted after capture of an electron with energy $w$ on an initial state $i$ leading to a final state $j$ is:

$$
E_{\nu}=m_{e} c^{2}\left(w+Q_{i j}\right)
$$

The neutrino spectra for a specific nuclear transition $i \rightarrow j$ is given by the respective partial rate per energy interval. The total spectrum is then the sum over all possible transitions. The normalized neutrino spectrum $n\left(E_{\nu}\right)$ is obtained by dividing by the total electron capture rate.

In the derivation above we have explicitly assumed that the neutrinos produced by the electron capture process leave the star; i.e. there is no blocking of the phase space due to the presence of neutrinos in the stellar environment. The spectra, which we will discuss below, are all derived based on this assumption. In the collapse phase following the presupernova evolution neutrinos are getting increasingly trapped in the core. This will require the inclusion of a neutrino blocking factor $\left(1-S_{\nu}\left(E_{\nu}\right)\right)$ in the phase space integral of the electron capture formula, Eq. (1). The neutrino distribution $S_{\nu}\left(E_{\nu}\right)$ depends on position and time and can be calculated within the Boltzmann transport formalism. Once $S_{\nu}\left(E_{\nu}\right)$ is known, the present neutrino spectra and the capture rates of 10 can be easily corrected for neutrino blocking. For the neutrino spectra this is achieved by folding the uncorrected spectra $n\left(E_{\nu}\right)$, as presented in this paper, with the blocking factor $\left(1-S_{\nu}\left(E_{\nu}\right)\right)$. The corrected capture rates are obtained by multiplying the tabulated rates 11] with $\int n(E)\left(1-S_{\nu}(E)\right) d E$.

For the following discussion it is useful to realize that the neutrino spectra depend basically on three quantities

- the electron chemical potential

- the 'effective' Q-value, $Q_{\text {eff }}=M_{p}-M_{d}+E_{i}$

- the GT strength distribution.

Generally one expects that neutrino energies are increased for larger electron chemical potentials, favorable mass differences between daughter and parent nuclei $\left(M_{p}-M_{d}\right)$, and from thermally excited states. Furthermore, large neutrino energies are favored if the strong GT transition resides at low excitation energies in the daughter nucleus. Due to nuclear pairing structure arguments 10] this is the case for odd-odd daughter nuclei (capture on even-even parents), while the bulk of the GT strength is somewhat higher $(\sim 2-3 \mathrm{MeV})$ for odd-A nuclei and is shifted by additional $2-3 \mathrm{MeV}$ in even-even daughter (capture on odd-odd parents) [12]. We note that these differences in the GT distributions result in the fact that low-lying rather weak GT transitions contribute relatively more to the electron capture rates on odd-odd nuclei and odd-A nuclei, while for even-even parents the GT bulk often resides at such low energies (e.g. $\mathrm{Ni}$ isotopes) that a distiction between low-lying strength and GT bulk is not very meaningful.

To illustrate the discussion we plot in Fig. 1 the normalized neutrino spectra for electron capture on the $\mathrm{A}=56$ isobars. The calculation has been performed for typical conditions during silicon shell burning of a $15 \mathrm{M}_{\odot}$ star [2] $\left(T=4 \times 10^{9} \mathrm{~K}, \rho=3 \times 10^{8} \mathrm{~g} \mathrm{~cm}^{-3}, Y_{e}=0.45\right)$. The resulting electron chemical potential then is $\mu_{e}=2.5$ $\mathrm{MeV}$.

We note that ${ }^{56} \mathrm{~V},{ }^{56} \mathrm{Cr},{ }^{56} \mathrm{Mn}$ and ${ }^{56} \mathrm{Fe}$ have $Q_{0}=$ $M_{p}-M_{d}<0$, thus making electron capture in the laboratory impossible. The other two nuclei ${ }^{56} \mathrm{Co}\left(Q_{0}=4.06\right.$ $\mathrm{MeV})$ and ${ }^{56} \mathrm{Ni}\left(Q_{0}=1.62 \mathrm{MeV}\right)$ decay dominantly by electron capture. With the exception of ${ }^{56} \mathrm{Co}$ the neutrino spectra for the other 5 nuclei are very similar: they are peaked around rather small neutrino energies $E_{\nu} \approx 1-2 \mathrm{MeV}$ with a width of $1.4-1.8 \mathrm{MeV}$. The reason for this quite similar structure is twofold. For the nuclei with negative $Q_{0}$-values, electron capture is hindered and requires electrons from the exponentially decreasing tail of the FD distribution. Obviously to achieve an appreciable rate it is advantegeous to keep the neutrino energies 


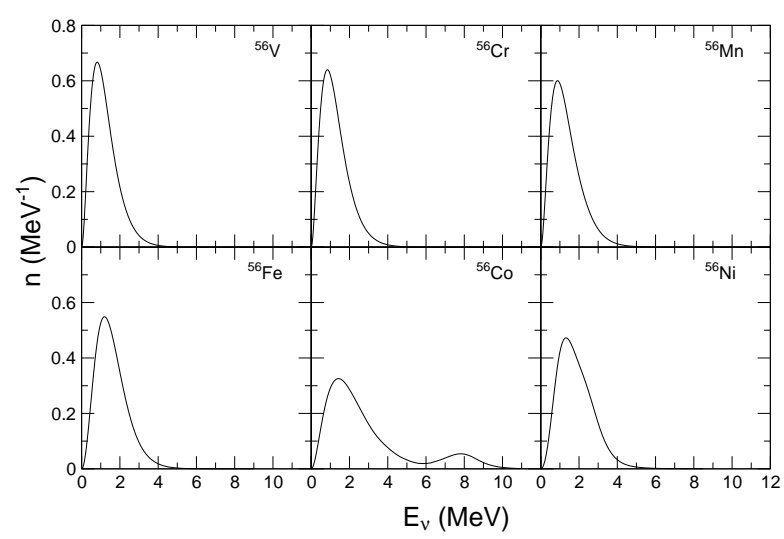

FIG. 1. Normalized neutrino spectra for stellar electron capture on selected $A=56$ isobars. The spectra have been calculated for stellar conditions which are typical for silicon shell burning in a $15 \mathrm{M}_{\odot}$ star [2].

small. Thus capture to low-lying states occurs with electrons with lower energies than capture to the bulk of the GT strength, but both are accompanied by low-energy neutrinos. For ${ }^{56} \mathrm{Ni}$ the $Q_{0}$-value allows capture of electrons with all energies. However, the GT distribution in the daughter ${ }^{56} \mathrm{Co}$ is well concentrated at low excitation energies, resulting again in a rather narrow neutrino spectrum.

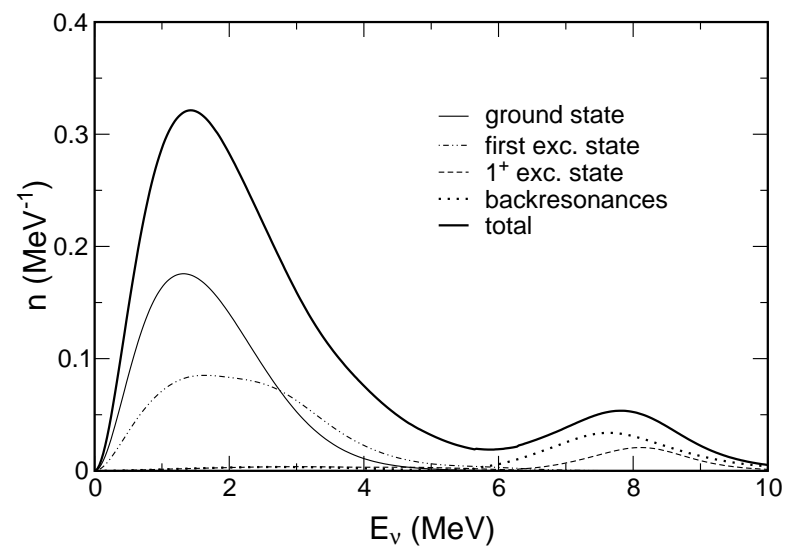

FIG. 2. Partial contributions of individual states in the parent nucleus to the neutrino spectrum for stellar electron capture on ${ }^{56}$ Co. The calculation has been performed for the same conditions as in Fig. 1. The spectra are multiplied by their relative weight to the total capture rate.

The ${ }^{56}$ Co spectrum is quite different, showing a doublebump structure. The reason is explained in Fig. 2 which shows the partial neutrino spectra contributed by selected states in the parent nucleus. The spectra for both, the ground state $(J=4)$ and the first excited state $(J=3)$ show the single-peak structure, as generally observed for the other nuclei. We also find the spectra for the first excited state somewhat wider than for the ground state. This reflects the strong angular momentum mismatch for the ground state which does not connect to states in ${ }^{56} \mathrm{Fe}$ below $2 \mathrm{MeV}$ and has only very weak transitions to states below $3 \mathrm{MeV}$. This is different for the excited state which can reach the lower-lying $J=2$ states in ${ }^{56} \mathrm{Fe}$ and in that way produces neutrinos with larger energies. Interestingly the excited $J=1$ state at $1.7 \mathrm{MeV}$ excitation energy in ${ }^{56} \mathrm{Co}$ produces a neutrino spectrum with a peak energy around $E_{\nu}=8$ $\mathrm{MeV}$. We note that this state has a rather strong GT matrix element to the ${ }^{56} \mathrm{Fe}$ ground state and hence, considering its favorable effective $\mathrm{Q}$ value of $Q_{\text {eff }} \sim 5.8 \mathrm{MeV}$, electron capture on this state generates neutrinos with rather large energies. While the excitation energy in the parent increases the effective Q-value, and thus the average neutrino energy, it reduces the contribution to the rate due to the Boltzmann weight. However, the excited $1^{+}$state yields the clue to the higher-energy neutrino peak. We notice that there are many more states in the excitation energy range $\sim 2-4 \mathrm{MeV}$ which are connected to the low-lying states in the daughter nucleus ${ }^{56} \mathrm{Fe}$ by strong GT transitions. FFN have coined the term 'backresonances' for these states [8,9] as they are part of the bulk of the GT strength built on the low-lying states in the inverse direction (Ref. [10] explains how these states are considered in the rate evaluation.) Electron capture on these backresonances occurs with favorable $Q_{\text {eff-value }}$ and hence allows the emission of neutrinos with rather high energies. Despite the Boltzmann suppression the gain in phase space combined with the large matrix elements ensure that these states combined contribute noticeably to the total rate and produce the second peak in the total neutrino spectrum. Noting that the integral over the spectrum reflects the relative contribution to the total rate, we remark in passing that Fig. 2 also implies that at the chosen conditions electron capture on ${ }^{56} \mathrm{Co}$ is dominated by the one on the ground state. Although similar in nuclear structure, the electron capture on the odd-odd nuclei ${ }^{56} \mathrm{Mn}$ and ${ }^{56} \mathrm{~V}$ does not produce a double-bump structure due to the negative $Q_{0}$ value which favors emission of low-energy neutrinos.

At the conditions of silicon shell burning, depicted in Fig. 2, the electron chemical potential is yet not large enough $\left(\mu_{e}=2.5 \mathrm{MeV}\right)$ to allow significant capture on the odd-odd nucleus ${ }^{56} \mathrm{Co}$ from low-lying states to the bulk of the $\mathrm{GT}_{+}$distribution in the daughter nucleus which resides at around $\sim 7-9 \mathrm{MeV}$ in ${ }^{56} \mathrm{Fe}[16]$. However, in the subsequent stellar evolution $\mu_{e}$ increases rather fast. Thus, capture to the bulk, e.g. with significantly larger GT strength, becomes easier for the lowlying states. This increases their relative weight compared with the one of the backresonances and is also not compensated by the relative gain in the Boltzmann factor of the latter. This behavior is demonstrated in Fig. 3, again for ${ }^{56} \mathrm{Co}$. The temperatures have been chosen accordingly, using the stellar trajectories as given by Heger et al. 2. We also observe that, once capture to the bulk of the GT distribution dominates the capture rate, 


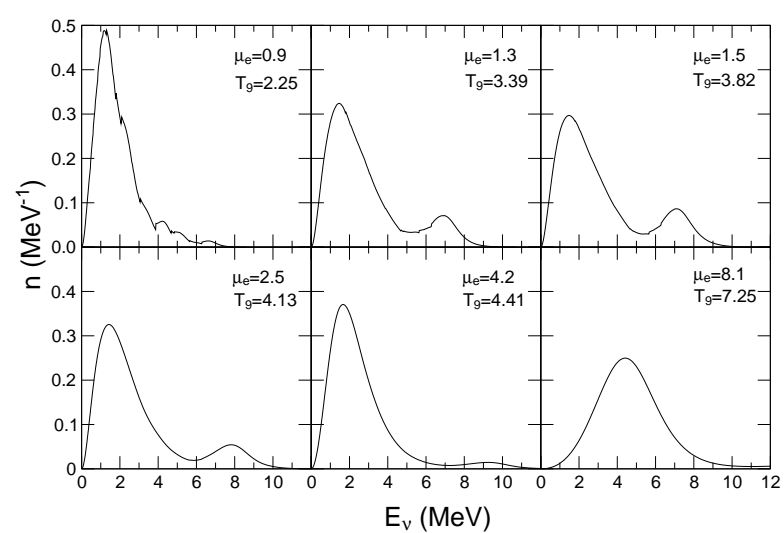

FIG. 3. Normalized neutrino spectra for stellar electron capture on ${ }^{56} \mathrm{Co}$ at several different phases of the final evolution of a $15 M_{\odot}$ star. The stellar parameters have been taken from Table 1 of 2 . The chemical potentials are given in $\mathrm{MeV}$, while $T_{9}$ defines the temperature in $10^{9} \mathrm{~K}$.

the spectrum becomes single-peaked (approximately for $\left.\mu_{e}=4.2\right)$. A further increase in $\mu_{e}$ then simply increases the average neutrino energy (e.g. for $\mu_{e}=8.1$ ).

We summarize that the relative height of the two peaks reflects the competition between electron chemical potential (its decrease reduces the capture from low-lying states to the bulk of the GT distribution) and temperature (its decrease reduces the Boltzmann weight of the backresonances). If one follows the stellar evolution backwards in time, i.e. to smaller temperatures, densities and electron chemical potential, the spectrum ultimatively becomes single-peaked as it is dominated by the temperature-favored ground state contribution. The spectrum also develops 'discontinuities' (see e.g. for $\mu_{e}=$ 0.9 ) which reflect the fact that the spectrum represents a sum over several initial and final states which all have a definite minimal neutrino energy $E_{\nu}^{\min }=m_{e} c^{2}\left(Q_{i j}+1\right)$. At $E_{\nu}^{\text {min }}$, the electron momentum vanishes $(p=0)$, but $p F(z, \omega)$ is finite resulting in a finite value for $n\left(E_{\nu}^{\min }\right)$. For larger $\mu_{e}$ values these discontinuities are smeared out as the individual spectra noticeably overlap.

As examplified for ${ }^{56} \mathrm{Fe}$ in Fig. 4 the situation is quite different if one studies the neutrino spectrum emerging from capture on an even-even nucleus as function of electron chemical potential. To understand the reason we note two facts. First, as the GT distribution in the daughter is quite concentrated at low excitation energies a double-peak structure related to the distinct capture to low-lying states and the GT bulk does not emerge. Second, in even-even nuclei the backresonances are at higher excitation energies than in odd-odd nuclei [10]. The consequences for the neutrino spectrum are obvious. If compared to ${ }^{56} \mathrm{Co}$, the relative contribution of the low-lying states to the electron capture on ${ }^{56} \mathrm{Fe}$ is significantly enhanced with respect to the backresonances and no highenergy neutrino peak emerges. As an increase of the

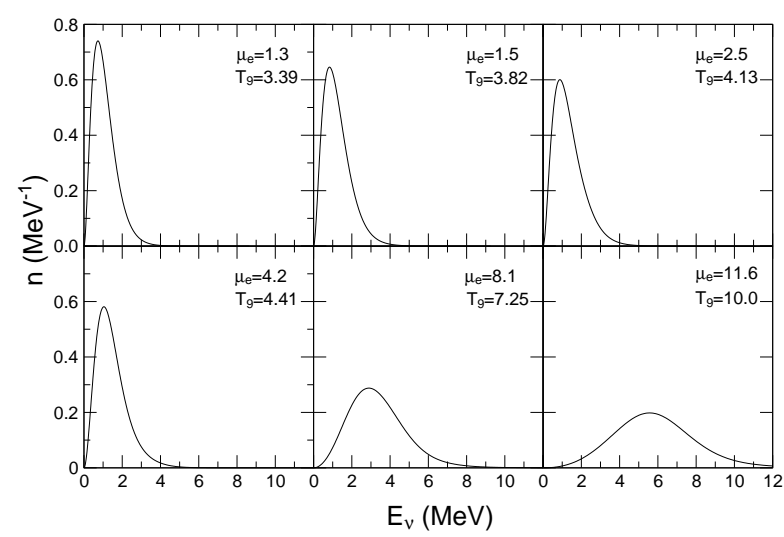

FIG. 4. Normalized neutrino spectra for stellar electron capture on ${ }^{56} \mathrm{Fe}$ at several different phases of the final evolution of a $15 \mathrm{M}_{\odot}$ star. The stellar parameters have been taken from Table 1 of [2]. The last panel corresponds to typical conditions during the collapse phase $\left(T=10^{10} \mathrm{~K}, \rho=3 \times 10^{10}\right.$ $\mathrm{g} / \mathrm{cm}^{3}$, and $\left.Y_{e}=0.42\right)$. The chemical potentials are in $\mathrm{MeV}$, while $T_{9}$ defines the temperature in $10^{9} \mathrm{~K}$.

electron chemical potential makes the capture energetically easier, the neutrino peak energy moves to higher energies. We mention that the last panel of the figure already corresponds to a phase of the contraction after the presupernova model which we have approximated by $T=10^{10} \mathrm{~K}, \rho=3 \times 10^{10} \mathrm{~g} / \mathrm{cm}^{3}$ and $Y_{e}=0.42$. It demonstrates that the simple structure of the spectrum remains also during that stellar evolution stage which requires detailed neutrino transport.

As stated above detailed neutrino transport becomes important in the final evolution of massive stars, following the presupernova models. Due to electron captures the matter in the final presupernova models is neutronrich and the nuclei present have negative $Q_{0}$-values. On the other hand, the electron chemical potential has grown strongly enough until this point thus allowing capture to the bulk of the GT strength. Fig. 5 shows the neutrino spectra for the 6 nuclei which dominate electron capture in the presupernova models of a $15 \mathrm{M}_{\odot}$ star [2. Due to Heger et al. the core density and temperature are $\rho=9.1 \times 10^{9} \mathrm{~g} / \mathrm{cm}^{3}$ and $T=7.2 \times 10^{9} \mathrm{~K}$, while the $Y_{e}$ value is 0.432 [2]. The chemical potential then is $\mu_{e}=8.1 \mathrm{MeV}$. We note that all neutrino spectra are single-peaked. The average neutrino energy released by nuclei is about $3 \mathrm{MeV}$, while it is $6.25 \mathrm{MeV}$ for capture on free protons which under these presupernova conditions become abundant enough to significantly contribute to electron capture.

As neutrino cross sections scale with $E_{\nu}^{2}$, high-energy neutrinos are more easily trapped. Capture on free protons has a more favorable $Q_{0}$ value than capture on neutronrich nuclei present in the presupernova matter composition. As a consequence the neutrino average energy is higher for capture on free protons. Nevertheless captures on nuclei still produces the larger amount of high-energy 


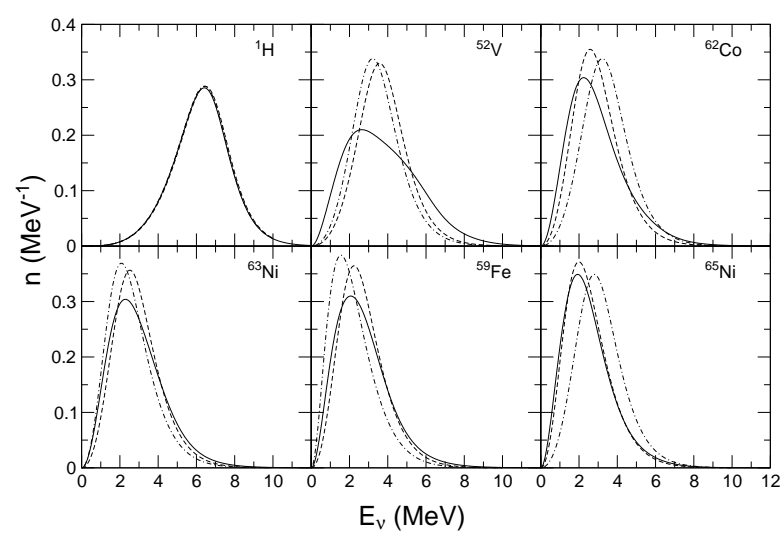

FIG. 5. Normalized neutrino spectra for stellar electron capture on the six most important 'electron-capturing nuclei' in the presupernova model of a $15 \mathrm{M}_{\odot}$ star, as identified in 22. The stellar parameters are $T=7.2 \times 10^{9} \mathrm{~K}$, $\rho=9.1 \times 10^{9} \mathrm{~g} / \mathrm{cm}^{3}$, and $Y_{e}=0.43$. The solid lines represent the spectra derived from the shell model electron capture rates. The dashed line shows the fit to the spectra, using the parametrization of Eq. (6) and adjusting the parameter $q$ to the average neutrino energy of the shell model spectrum. The dashed-dotted spectrum corresponds to the parametrization recommended in 河.

neutrinos in lighter stars $\left(\mathrm{M}<20 \mathrm{M}_{\odot}\right)$ as they dominate the rate in the presupernova models. This is different for heavier stars where the capture on free protons in the presupernova model corresponds already to $30-50 \%$ [2].

For presupernova conditions the shell model evaluation predicts slightly larger average neutrino energies than the FFN rates [2]. A possible explanation for this difference is given by the fact that the shell model rates explicitly consider capture from thermally excited states. The Qvalue for ${ }^{59} \mathrm{Fe}$ is $Q_{0}=-5.696 \mathrm{MeV}$. However, the excited states have more favorable $Q_{\text {eff values than the ground }}$ state thus making electron capture more easy and in several cases supporting larger neutrino energies. Fig. 6 shows the neutrino spectrum calculated for the 6 lowest states in ${ }^{59} \mathrm{Fe}$. (Here the distributions are multiplied by their relative weight in the total rate). One finds that indeed neutrino spectra from excited states often have a wider tail. This results from capture to low-lying states in the daughter. In contrast to the low-lying transitions the GT bulk approximately obeys Brink's hypothesis, e.g. [17]. This states that the GT strength of excited states is the same as for the ground state, only shifted by the excitation energy of the parent state. In particular, Brink's hypothesis implies that the relevant energy difference $Q_{\mathrm{eff}}-E_{j}$ is the same for capture to the GT bulk for all parent states. Another observation has already been mentioned above. Although the total capture rate is still dominated by the transition to low-lying states, the main source for high-energy neutrinos, however, are the 'backresonances' 8, 8 .

If the shell model neutrino spectra are to be used
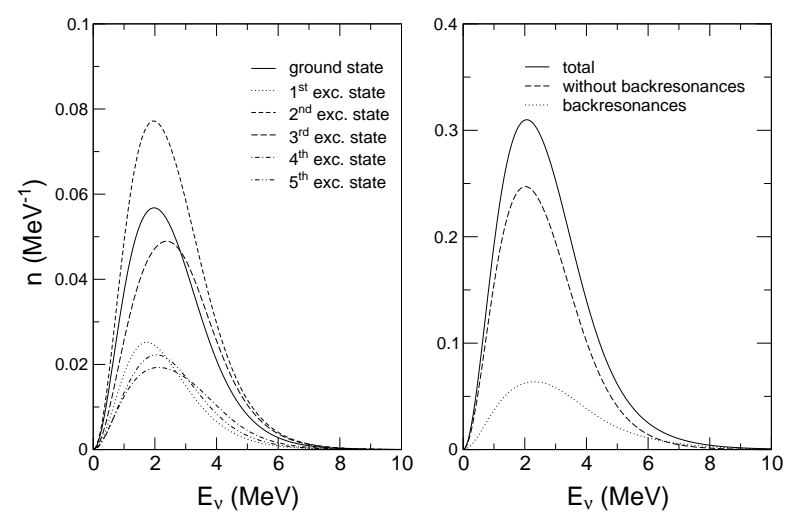

FIG. 6. Partial contributions of individual states in the parent nucleus to the Neutrino spectrum for stellar electron capture on ${ }^{59} \mathrm{Fe}$. The calculation has been performed for the presupernova conditions of Fig. 5. The spectra are multiplied by their relative weight to the total capture rate. The left panel shows the neutrino spectra calculated for the ground state (solid line) and the 5 lowest excited states in ${ }^{59} \mathrm{Fe}$. The right panel compares the total neutrino spectrum (solid line) with the one obtained only from the backresonances (dotted line, for a definition see text and 10]). The dashed line shows the contributions from the low-lying individual states which have been considered to calculate the electron capture rate [10.

in collapse simulations, they have to be represented by parametrizations which are accurate, fast and can be easily implemented. Our proposal for the parametrization is based on the following approximations. Suppose that i) the electron capture on a state in the parent nucleus, described by GT transitions, leads to a single state in the daughter nucleus at energy $E_{f}^{*}$ and that ii) Brink's hypothesis is valid, i.e. this state is at $E_{f}^{*}+E_{i}$ if the capture is on an excited state in the parent at excitation energy $E_{i}$. If we equal electron energy and momentum, which is a valid approximation for the conditions we are interested in, the neutrino spectrum has the form [5]

$$
n\left(E_{\nu}\right)=E_{\nu}^{2}\left(E_{\nu}-q\right)^{2} \frac{N}{1+\exp \left\{\left(E_{\nu}-q-\mu_{e}\right) / k T\right\}}
$$

with $q=Q_{0}-E_{f}^{*}$ and a constant $N$ which normalizes the neutrino spectrum to unity. This form is obviously valid for capture on free protons where the parameter $q$ is the reaction $Q_{0}$ value, $q=-1.29 \mathrm{MeV}$. For finite nuclei, $q$ should be considered a fit parameter. It can be adjusted to the average neutrino energy which is listed in the shell model rate tabulations [11] for a grid of temperature/density points and can be easily interpolated in-between. We have tested this proposal and generally find that the parametrization approximates the shell model spectra rather well, as can be seen by the dashed curves in Fig. 5. Of course, our parametrization fails if the spectrum is double-peaked as observed for capture on odd-odd nuclei under special conditions (e.g. see Fig. 
1.). However, we do not expect that these conditions occur for $p f$-shell nuclei during the collapse phase where the electron chemical potential is high enough compared to the reaction $Q_{0}$ value to allow appreciable electron capture to the GT bulk. Finally we note that for ${ }^{52} \mathrm{~V}$ the shell model spectrum is wider than the parametrization. This is caused by the fact that the spectrum for the individual states shows noticeable differences and does not strictly follow the Brink hypothesis. For example, the excited state of ${ }^{52} \mathrm{~V}$ at $22 \mathrm{keV}$ has $J=5$ and thus there are no low-lying states in the daughter ${ }^{52} \mathrm{Ti}$ which can be reached by GT transitions. This is different for the excited $J=1$ state at $141 \mathrm{keV}$ which connects strongly to the ${ }^{52} \mathrm{Ti}$ ground state.

Bruenn suggested a similar parametrization for the neutrino spectra emerging from electron capture on $p f$ shell nuclei, however simply setting $q=Q_{0}-3 \mathrm{MeV}[5]$. The resulting spectra are compared to the shell model spectra in Fig. 5. Despite the simple guess for the parameter, the agreement is quite acceptable.

In summary, the knowledge of the neutrino energy spectra at every point and time in the core is quite relevant for simulations of the final collapse and explosion phase of a massive star. In the collapse phase, neutrinos are mainly produced by electron capture on nuclei and protons and their emerging energy spectra are an important ingredient in the simulations. In this paper we have presented neutrino spectra for stellar electron capture during the final presupernova evolution stage of massive stars. The spectra have been consistently derived in the framework of the recently evaluated capture rates which have been calculated on the basis of state-of-theart large-scale shell model studies. Furthermore we have calculated the spectra for stellar conditions which have been obtained in presupernova evolution of massive stars, using the same shell-model weak interaction rates. The calculated presupernova neutrino spectra show a rather simple structure which is easily parametrizable. This parametrization is easily implementable into the simulation codes and allows for a derivation of the neutrino spectra consistent with the shell-model weak-interaction rates.

\section{ACKNOWLEDGMENTS}

Our work has been supported by the Danish Research Council. GMP thanks the Carlsberg Foundation for a fellowship. JMS acknowledges a scholarship of the Fundação para a Ciência e Tecnologia.
[1] T.A. Weaver, S.E. Woosley and G.M. Fuller, in Numerical Astrophysics, eds. J. Centrella, J. LeBlanc and R. Bowers (Jones and Bartlett, 1984) p. 374

[2] A. Heger, S. E. Woosley, G. Martínez-Pinedo and K. Langanke, submitted to Astrophys. J. , astro-ph/0011507

[3] S.E. Woosley and T.A. Weaver, Astrophys. J. Suppl. Ser. 101, 181 (1995)

[4] A. Mezzacappa, Nuclei in the Cosmos, eds. J. Christensen-Dalsgaard and K. Langanke, Nucl. Phys. A, to be published

[5] S.W. Bruenn, Astrophys. J. Suppl. Ser. 58, 771 (1985)

[6] A. Mezzacappa and S.W. Bruenn, Astrophys. J. 410, 740 (1993)

[7] H.-T. Janka and E. Müller, Astron. Astrophys. 306, 167 (1996)

[8] G.M. Fuller, W.A. Fowler and M.J. Newman, Astrophys. J. Suppl. Ser. 42, 447 (1980)

[9] G.M. Fuller, W.A. Fowler and M.J. Newman, Astrophys. J. Suppl. Ser. 48, 279 (1982)

[10] K. Langanke and G. Martínez-Pinedo, Nucl. Phys. A 673, 481 (2000)

[11] K. Langanke and G. Martínez-Pinedo, At. Data and Nucl. Data Tables, in print

[12] E. Caurier, K. Langanke, G. Martínez-Pinedo, and F. Nowacki, Nucl. Phys. A 653, 439 (1999)

[13] A. Heger, K. Langanke, G. Martínez-Pinedo and S.E. Woosley, Phys. Rev. Lett. in print, astro-ph/0007412

[14] H.A. Bethe, G.E. Brown, J. Applegate and J.M. Lattimer, Nucl. Phys. A 324, 487 (1979)

[15] I.S. Towner and J.C. Hardy, in: Symmetries and Fundamental Interactions in Nuclei, eds. W.C. Haxton and E.M. Henley (World Scientific, Singapore, 1995) p. 183

[16] K. Langanke and G. Martínez-Pinedo, Phys. Lett. B 453, 187 (1999)

[17] M.B. Aufderheide, I. Fushiki, S.E. Woosley, D.H. Hartmann, Astrophys. J. Suppl. Ser. 91, 389 (1994) 